\title{
The rheology of solid glass
}

\author{
Stephen R. Williams ${ }^{\text {a) }}$ and Denis J. Evans \\ Research School of Chemistry, The Australian National University, Canberra, \\ Australian Capital Territory 0200, Australia
}

(Received 12 November 2009; accepted 7 April 2010; published online 12 May 2010)

\begin{abstract}
As the glass transition is approached from the high temperature side, viewed as a liquid, the properties of the ever more viscous supercooled liquid are continuous functions of temperature and pressure. The point at which we decide to classify the fluid as a solid is therefore subjective. This subjective decision does, however, have discontinuous consequences for how we determine the rheological properties of the glass. We apply the recently discovered relaxation theorem to the time independent, nondissipative, nonergodic glassy state to derive an expression for the phase space distribution of an ensemble of glass samples. This distribution is then used to construct a time dependent linear response theory for aged glassy solids. The theory is verified using molecular dynamics simulations of oscillatory shear for a realistic model glass former with excellent agreement being obtained between the response theory calculations and direct nonequilibrium molecular dynamics calculations. Our numerical results confirm that unlike all the fluid states, including supercooled liquids, a solid glass (in common with crystalline states) has a nonzero value for the zero frequency shear modulus. Of all the states of matter, a supercooled fluid approaching the glass transition has the highest value for the limiting zero frequency shear viscosity. Finally, solid glasses like dilute gases and crystals have a positive temperature coefficient for the shear viscosity whereas supercooled and normal liquids have a negative temperature coefficient. () 2010 American Institute of Physics. [doi:10.1063/1.3418442]
\end{abstract}

\section{INTRODUCTION}

A fundamental understanding of the glass transition is a long standing question in condensed matter physics. ${ }^{1,2}$ If the glass transition is approached by lowering the temperature of a liquid while preventing crystallization, then it is easy to view a glass as an extremely viscous liquid. The viscosity increases continuously, apparently without limit, with correlation times diverging toward infinity. ${ }^{2}$ There has been considerable work on the rheology and flow of glass from the point of view of liquid state theory (e.g., see Refs. 2-7 and references therein). However, if we take a glass sample well below the glass transition temperature and allow it to age, then macroscopically, it appears like any other solid material with a nonzero shear modulus. On the human time scale it appears as though the glass can support a shear stress indefinitely. Obsidian, a naturally occurring glass, does not flow even on a timescale of a hundred million years. The stress relaxation time $\tau_{M}$ has diverged effectively to infinity which, in reality, means that the Deborah number (the ratio of stress relaxation time to maximum available observation time $D_{e}$ $\equiv \tau_{M} / \tau_{\text {obs }}$, see Ref. 8) has diverged.

Because the glass transition is continuous, it ultimately becomes a subjective decision as to precisely where the glass transition temperature $T_{g}$ is; below this temperature, we regard the glass as solid and above it we regard the system to be a liquid. This decision will be strongly influenced by the Deborah number. As we approach $T_{g}$ from higher tempera-

${ }^{\text {a)} E l e c t r o n i c ~ m a i l: ~ s w i l l i a m s @ ~ @ r s c . a n u . e d u . a u . ~}$ tures, the Deborah number will grow at a very strongly increasing rate; so much so that the precise value of $T_{g}$ will not be overly sensitive to our subjective choice.

From the liquid state point of view, a glass is often $d e$ fined to be a supercooled liquid with a viscosity greater the $10^{13}$ poise. $^{2}$ However, if we as humans lived on a geological rather than biological timescale, we might raise that threshold viscosity significantly. In fact, a glass well below the glass transition temperature is not terribly different from a crystalline solid below its melting point. At any finite temperature, the motion of defects, grain boundaries, dislocations, etc., mean that on some enormously long timescale, all crystalline solids will flow in response to a static stress. It has a finite (but exceedingly large!) shear viscosity when viewed as an exceedingly slow fluid.

The time it takes for a liquid to relax to equilibrium after a quench will be strongly influenced by the stress relaxation time. At or close to $T_{g}$, the stress relaxation time will be large, such that the Deborah number may have a value of unity or so, and the equilibration time may well exceed the observation time. In this region one may observe significant flow or creep over the longest available observation time scale. Such systems, left undisturbed, simply fail to relax to equilibrium - they "creep" even over the longest available timescales. ${ }^{9-12}$ Because of this inherent creep, the response of such a system to a periodic shear will not be periodic and it will not be possible to characterize the material in terms of a complex frequency dependent viscosity or storage and loss modulus. The typical phenomenological viscoelastic treatment ${ }^{13}$ of liquids or solids will fail for these materials. The material properties slowly change in time as a reflection 
of the underlying creep that is constantly taking place. Here we will not consider creep and related phenomena further.

This leaves us with the case of a glass that has been aged sufficiently that it does not evolve or flow significantly on the observation time scale. Ordinary window pane glass is a perfect example. For such a glass, the Deborah number is effectively infinite $D_{e}=\lim \left(\tau_{M} \rightarrow \infty\right) \tau_{M} /\left[\tau_{\text {obs }} \rightarrow \infty\right]$, and the glass will be out of thermodynamic equilibrium with its properties depending on its preparative history. As Landau and Lifshitz ${ }^{14}$ point out, a glass is the limiting case of a viscoelastic fluid where the relaxation time and therefore the shear viscosity have become infinite.

Assuming a simple Maxwell model for a viscoelastic supercooled fluid where the $x y$ element of the frequency dependent pressure tensor $\widetilde{P}_{x y}(\omega)$ is related to the frequency dependent strain rate tensor $\tilde{\dot{\gamma}}(\omega)$ by the equation $\widetilde{P}_{x y}(\omega)=$ $-\tilde{\eta}(\omega) \tilde{\dot{\gamma}}=-G \tau_{M} \tilde{\dot{\gamma}} /\left(1+i \omega \tau_{M}\right)$, where $\tilde{\eta}(\omega)$ is the complex frequency dependent shear viscosity, a solid glass is obtained in the $\operatorname{limit} \lim \left(\tau_{M} \rightarrow \infty\right) \widetilde{P}_{x y}(\omega)=i G \tilde{\dot{\gamma}} / \omega=-G \tilde{\gamma}$. This equation is the constitutive relation for an ideal elastic solid under a frequency dependent strain $\tilde{\gamma}$. For fluids, $G$ is equal to the infinite frequency shear modulus. For solid glasses, in this limit, under this simple minded Maxwell model, $G$ becomes the frequency independent shear modulus of the ideal solid glass.

Before continuing the discussion, we should mention that as the Maxwell relaxation time diverges to infinity so does the time taken to relax to equilibrium. This in turn means that in this limit, we expect a history dependent residual shear stress $P_{x y \text {,res }}$, to also be present, $\lim \left(\tau_{M} \rightarrow \infty\right) \tilde{P}_{x y}(\omega)=-G \tilde{\gamma}+P_{x y, \text { res }} / i \omega$.

Now of course there is no such thing as an ideal elastic solid. No such solid exits in Nature. The shear modulus must show dispersion (i.e., it must show frequency dependence). In fact dispersion also implies that there must be dissipation because the two are related through the Kramers-Kronig relation. ${ }^{15}$ Any variation in the real part of the shear modulus with frequency, implies that there has to be dissipation. Dissipation characterizes the rate at which work done by shearing a system is converted irreversibly into heat. The dissipation generated by a low frequency shearing deformation is characterized by the solid's limiting zero frequency shear viscosity which is neither divergent nor zero. Landau and Lifshitz ${ }^{14}$ use the terms "internal friction" and "solid viscosity" for what we refer to as the limiting zero frequency viscosity. We only treat the case of shearing deformations whereas Landau and Lifshitz treat shear and bulk deformations. Of course, if we do apply a constant strain rate to a solid, once the strain exceeds $\sim 0.1$, some sort of inelastic behavior will occur (fracture or plastic flow). So, in fact, the zero frequency shear viscosity cannot be defined for a solid. There is a very old history of experimental work on measuring dissipation in solids see the 1892 paper by Voight ${ }^{16}$ and the theoretical paper by Zener $^{17}$ in the late 1930s. Understanding dissipation in solids undergoing shearing deformations has recently had a revival of interest due to the importance of understanding damping of nano-oscillators. ${ }^{18}$

In spite of this problem at zero frequency, we can calcu- late the frequency dependent shear viscosity at any nonzero frequency. One simply needs to control the amplitude of the strain rate so that the amplitude of the strain stays less than the elastic response limit $(\sim 0.1)$ and then one can calculate the limiting zero frequency, $\lim (\omega \rightarrow 0)$, shear viscosity $\widetilde{\eta}\left(0^{+}\right) \equiv \lim (\omega \rightarrow 0) \tilde{\eta}(\omega)$. However, there is another important difference that occurs when treating solids rather than fluids. In the zero frequency limit for fluids, the stress is entirely dissipative. For solids which by definition have a finite zero frequency shear modulus $G_{0}$, only part of the limiting zero frequency stress is dissipative. The Newtonian constitutive relation even modified to work at small but finite frequencies does not apply to solids. For slowly changing strain rates, the constitutive relation for solid glasses reads $\lim (\omega \rightarrow 0)\left\langle P_{x y}(t)\right\rangle=-\tilde{\eta}\left(0^{+}\right) \dot{\gamma}(t)-G_{0} \gamma(t)+P_{x y \text {,res. }} \quad$ This, in turn, means the statistical mechanical expressions which relate stress averages and time correlation functions to transport coefficients and elastic moduli, also change for a solid. For glasses at low frequencies any residual quasiequilibrium stress must be removed from the Green-Kubo integrals. We recently showed for the first time ${ }^{19}$ how this should be done. This is analogous to the Green-Kubo expressions for the bulk viscosity of fluids where the equilibrium hydrostatic pressure must be removed to form the viscous pressure tensor for use in forming Green-Kubo relations.

We have verified using Green-Kubo relations and nonequilibrium molecular dynamics for a simple liquid and corresponding crystal, that either side of the freezing transition, the limiting zero frequency shear viscosity $\tilde{\eta}\left(0^{+}\right)$, for the crystal and the liquid differ by no more than an order of magnitude or so. ${ }^{19}$ In that same paper, we showed that for a crystal the zero frequency shear modulus is nonzero while for a fluid it is identically zero. Perhaps more surprisingly, the most often used expression for the infinite frequency shear modulus of a fluid $G_{\infty}^{f}=\beta V\left\langle P_{x y}^{2}\right\rangle$ where $V$ is the volume and $\beta=1 / k_{B} T$, is not valid for solids (including crystals) at nonzero temperatures.

At first it may seem impossible to bring statistical mechanics to bear on the type of glass under consideration. The only phase space distribution functions that can be expressed in simple closed form describe systems in thermodynamic equilibrium and glasses, as we have said are not in equilibrium! However our recent proof of the relaxation theorem ${ }^{20}$ confirms that although glasses are not in true thermodynamic equilibrium, the distribution of states within the subdomain of phase space explored by an individual glass sample, nevertheless, must be Boltzmann weighted. Furthermore because the Deborah number is effectively infinite, these subdomains must be robust with respect to small but finite shearing deformations. If they were not, the Deborah number would be finite contradicting our assumption. These two observations confirm that for an ensemble of samples, the phase space distribution must be given by our recently derived quasiequilibrium distribution with intrasubdomain weights being Boltzmann and the intersubdomain weights being history dependent, non-Boltzmann, and robust. ${ }^{21}$ This is related to the earlier work of Palmer. ${ }^{22}$

In the present paper, we will give a derivation of the quasiequilibrium distribution for glass states and then use 
this as the basis for constructing linear response theory for glass systems in which the Deborah number is effectively infinite. (Throughout the remainder of this paper we will refer to such systems as glass, while amorphous systems with Deborah numbers less than unity shall be referred to as supercooled or normal liquids. Systems with Deborah numbers close to unity will not be considered.) We will apply response theory to the case where the system is driven by planar, oscillatory shear. When applied to a glass that has a history dependence, the results of this theory are quite different from the corresponding results of standard linear response (or Green-Kubo) theory for fluids, including supercooled liquids above the glass transition. While here we only consider planar shear, generalizing this theory to other processes is straightforward.

We will use both equilibrium and nonequilibrium molecular dynamics to confirm the correctness of our response theory. Our generalization of linear response theory reduces to the ordinary and well known linear response theory when applied to an equilibrium liquid. When applied to a glass with an effectively infinite Deborah number, the same theory identifies it as a solid. As yet, there is no comparable theory for an aging glass with finite Deborah number that is exhibiting slow flow or creep, etc. A complete understanding of the dramatic slowing of the liquid state as the putative glass transition is approached is not enough, by itself, to fully understand how solid behavior emerges in the glass phase. The work presented here provides significant new microscopically based insight on the emergence of solid behavior in glasses, which cannot be gained from liquid state based theories alone. As this work makes use of the dramatic separation in time scales, which has been established in many previous studies, ${ }^{2,6}$ it is, however, complementary to the existing work. As we will see in detail, the statistical mechanics of solid glasses is very different from that for fluids, even supercooled fluids, and is also different from the statistical mechanics of crystalline states.

Comparing the qualitative rheology among glass, fluid and, crystalline systems by use of simulations, we find that just above the glass transition temperature, supercooled liquids are unique in having extremely high values for the limiting zero frequency shear viscosity. This confirms the validity of one commonly used definition of glasses. However, below the glass transition, glasses can behave similarly to crystals in having nonzero values for the zero frequency shear modulus and values of the limiting zero frequency shear viscosity $\widetilde{\eta}\left(0^{+}\right)$that are comparable to those of the corresponding triple point liquid. We also find that above the glass transition (viewed as fluids), supercooled liquids behave very similarly to normal liquids exhibiting a negative temperature coefficient for the viscosity. Below the glass transition where the Deborah number is effectively infinite, glasses like crystals and dilute gases, have a positive temperature coefficient for the limiting shear viscosity $\widetilde{\eta}\left(0^{+}\right){ }^{19}$

\section{THEORY}

Here we will closely follow the approach in our previous paper $^{19}$ on crystalline solids. However, we will generalize the theory presented there to handle the case of nonergodic solids, which are unable to undergo any significant linear creep on the maximum time scale of interest (i.e., the Deborah number is effectively infinite).

\section{A. The equations of motion}

As in Ref. 19, we use the standard isokinetic equations of motion which feature a synthetic thermostat under the condition that the total peculiar momentum $\sum_{i=1}^{N} \mathbf{p}_{i} \equiv \mathbf{p}=0$, is always zero.

The equations of motion are

$$
\begin{aligned}
& \dot{\mathbf{q}}_{i}=\mathbf{p}_{i} / m_{i}+\mathbf{C}_{i}(\boldsymbol{\Gamma}) \cdot \mathbf{F}_{e} \\
& \dot{\mathbf{p}}_{i}=\mathbf{F}_{i}(\mathbf{q})+\mathbf{D}_{i}(\boldsymbol{\Gamma}) \cdot \mathbf{F}_{e}-\zeta \mathbf{p}_{i} \\
& \zeta=\frac{\sum_{i=1}^{N} \mathbf{p}_{i} \cdot \mathbf{F}_{i}+\mathbf{p}_{i} \cdot \mathbf{D}_{i}(\boldsymbol{\Gamma}) \cdot \mathbf{F}_{e}}{\sum_{i=1}^{N} \mathbf{p}_{i} \cdot \mathbf{p}_{i}},
\end{aligned}
$$

where $\mathbf{q}_{i}$ and $\mathbf{p}_{i}$ are the position and peculiar momentum of the $i$ th particle, $\mathbf{q}$ is the $3 N$ dimensional vector of all the positions, $\boldsymbol{\Gamma}=(\mathbf{q}, \mathbf{p})$ is the $6 N$ dimensional phase space vector, $m_{i}$ is the mass of the $i$ th particle, $\mathbf{F}_{i}$ is the force on the $i$ th particle due to interactions with other particles, $\mathbf{F}_{e}$ is the external field which drives the system and is set to zero for equilibrium dynamics, $\mathbf{C}_{i}$ and $\mathbf{D}_{i}$ are second rank tensors which couple the system to the external field, and $\zeta$ is the thermostat multiplier which holds the peculiar kinetic energy of the system fixed.

The equations of motion for the system undergoing planar shear can be written as the so called SLLOD ${ }^{23}$ equations of motion with Eq. (1)

$$
\begin{aligned}
& \dot{\mathbf{q}}_{i}=\mathbf{p}_{i} / m_{i}+\mathbf{i} \dot{\gamma} q_{y i} \\
& \dot{\mathbf{p}}_{i}=\mathbf{F}_{i}-\mathbf{i} \dot{\gamma} p_{y i}-\zeta \mathbf{p}_{i} \\
& \zeta=\frac{\sum_{i=1}^{N} \mathbf{p}_{i} \cdot \mathbf{F}_{i}-\dot{\gamma} p_{x i} p_{y i}}{\sum_{i=1}^{N} \mathbf{p}_{i} \cdot \mathbf{p}_{i}},
\end{aligned}
$$

where $\mathbf{i}$ is the unit vector in the direction of the $x$ Cartesian axis, $p_{x i}$ is the $x$ component of $\mathbf{p}_{i}$, and $\dot{\gamma}=d u_{x} / d y$ is the strain rate where $u_{x}$ is the streaming velocity which is set along the direction i. Equation (2) is used in conjunction with LeesEdwards shearing periodic boundaries. ${ }^{23}$ The thermostatting terms $\left(-\zeta \mathbf{p}_{i}\right)$ fix the peculiar kinetic energy of the system. The adiabatic forms of these equations give an exact description of adiabatic shear flow arbitrarily far from equilibrium. When subject to the Gaussian isokinetic thermostat as in Eq. (2), these equations give an exact description of shear flow in the linear response regime. ${ }^{23}$

\section{B. The quasiequilibrium distribution function}

It is well known that aged glassy systems are nonergodic. When viewed as fluids, these systems are arrested by virtue of their very high shear viscosities and consequent slow stress relaxation times. The Deborah number of a sample characterizes the ratio of the slowest stress relaxation time to the longest available observation time. During aging 
from a melt, the phase space domain within which a glass sample is trapped slowly changes. Eventually, these changes become insignificant over the longest available observation time and the Deborah number is effectively infinite. This is what we refer to as an aged glass. A given sample of an aged glass is trapped in a time independent subdomain of phase space say, $D_{\alpha}$. The sample is assumed to be in contact with a heat reservoir but otherwise obeys Newton's equations of motion. Although a glass is arrested due to its high fluid viscosity, we can use the newly developed relaxation theorem $^{20}$ to argue that for a fully aged glass sample trapped in some fixed ergodic subdomain of phase space, the time independent relaxed phase space distribution function must take the form of the quasiequilibrium canonical distribution first derived in Ref. 21 and related to that in Ref. 22.

Without loss of generality we write the initial phase space distribution as

$$
f_{\alpha}(\boldsymbol{\Gamma}, 0)=f_{F, \alpha}(\boldsymbol{\Gamma})=\frac{\exp [-F(\boldsymbol{\Gamma})]}{\int_{D_{\alpha}} d \boldsymbol{\Gamma} \exp [-F(\boldsymbol{\Gamma})]_{\alpha}}, \forall \boldsymbol{\Gamma} \in D_{\alpha},
$$

where $F(\boldsymbol{\Gamma})$ is a real, single valued function that is an even function of the momenta. This ensures that the average velocity of atoms in the sample is zero; the velocities are therefore peculiar velocities. If we subject the sample to small strains, or small changes in pressure or temperature, each sample stays within the phase space domain $D_{\alpha}$. We say that the ergodic phase space subdomains are robust with respect to small strains and temperature changes. These domains must in fact be robust otherwise the Deborah number of the glass would not be effectively infinite. (The reason for this is that the internal stress of an aged glass sample is nonzero, in general. If the domain is not robust with respect to small strains, the sample will flow to relax the strain and the Deborah number therefore cannot be infinite.) This further implies that the system obeys the condition known as ergodic consistency and the time averaged dissipation function $\bar{\Omega}_{t}$

$$
\bar{\Omega}_{t} t \equiv \ln \left(\frac{f_{F, \alpha}(\boldsymbol{\Gamma}(0))}{f_{F, \alpha}(\boldsymbol{\Gamma}(t))}\right)-\int_{0}^{t} d s \Lambda(s),
$$

where $\Lambda \equiv \partial \dot{\boldsymbol{\Gamma}} / \partial \boldsymbol{\Gamma}=-(3 N-4) \zeta$ satisfies the Evans-Searles Fluctuation theorem $^{24}$

$$
\frac{p\left(\bar{\Omega}_{t}=A\right)}{p\left(\bar{\Omega}_{t}=-A\right)}=\exp [A t] .
$$

If the domains are not robust, the Deborah number is not effectively infinite and the Evans-Searles fluctuation relation and the Crooks fluctuation relation ${ }^{25}$ cannot be satisfied. Thus, there are simple tests that are available to test domain robustness and therefore check whether the Deborah number is effectively infinite. ${ }^{21}$ Obviously, robustness is not always satisfied. Domains cannot be expected to maintain robustness near the glass transition temperature. Indeed, for glasses close to the glass transition, the present theory cannot be applied.

Thus far we have discussed phase space distribution functions and equations of motion but have said little about coordinate systems. In fluid mechanics, two main systems of coordinates are used: Eulerian or laboratory coordinate systems and Lagrangian or comoving coordinate systems. The fluctuation theorem works for both types of coordinate systems but the condition of ergodic consistency will apply more generally to problems written in Lagrangian coordinate systems. In this paper, we will talk about subjecting glasses to elastic deformations. In this case, domain robustness requires that the deforming domains described in Lagrangian or comoving coordinates are robust. If the deformation is reversed the domains return to their original Eulerian topology.

The system also obeys the dissipation theorem ${ }^{26,27}$ which states that the time dependent $\mathrm{N}$-particle distribution function can be written in terms of the time integrated dissipation function

$$
f_{\alpha}(\boldsymbol{\Gamma}(0), t)=\exp \left[-\int_{0}^{-t} d s \Omega(\boldsymbol{\Gamma}(s))\right] f_{\alpha}(\boldsymbol{\Gamma}(0), 0)
$$

This expression is valid arbitrarily far from equilibrium in the linear and nonlinear regimes, regardless of the initial state. It does not require either ergodic consistency or domain robustness. If we consider applying at time zero some small but finite perturbation to an already fully aged glass, we can use Eq. (6) for the cases of a system relaxing toward quasiequilibrium from an initial nonequilibrium distribution, or for the case of a system which is driven away from its initial quasiequilibrium distribution by an external field. If we wish to use an external field, then $\Omega(\boldsymbol{\Gamma}(s))$ must be determined using the equations of motion in the presence of the field and thermostat.

Thus, Eq. (6) shows that if the phase space distribution is time independent, with an effectively infinite Deborah number, then the dissipation function must be zero everywhere within the phase space subdomain $D_{\alpha \cdot}{ }^{20}$ From the definition of the dissipation function, it is trivial to see that if the dissipation function is zero everywhere in $D_{\alpha}$ and since this domain is robust because the Deborah number is effectively infinite, then the probability of observing any set of phase space trajectories is identical to the probability of observing the conjugate set of antitrajectories. All properties of the sample are invariant with respect to time reversal. This is what one would expect in a glass which has aged to the point where no significant further relaxation can occur on the longest time scale available for observation. We call such an ensemble a quasiequilibrium ensemble. It is not in true thermodynamic equilibrium because it is nonergodic over the full phase space and is trapped inside the phase space domain $D_{\alpha}$. This trapping prevents the system from exploring most of the phase space, including those areas corresponding to crystalline states, on the longest accessible time scale.

The thermostat fixes the peculiar kinetic energy $K_{\text {th }}$ $=K_{0}$ and the total peculiar momentum is a constant of the motion $\mathbf{p}_{\text {th }}(t)=0, \forall t$. It is straightforward to show (see Ref. 20 for details) that for our equations of motion with zero shear rate, the canonical subdomain distribution function 


$$
\begin{aligned}
& f_{\alpha}^{c}(\boldsymbol{\Gamma})=\frac{\exp \left[-\beta H_{0}(\boldsymbol{\Gamma})\right] \delta(\mathbf{p}) \delta\left(K_{\mathrm{th}}-K_{0}\right)}{\int_{D_{\alpha}} d \boldsymbol{\Gamma} \exp \left[-\beta H_{0}(\boldsymbol{\Gamma})\right] \delta(\mathbf{p}) \delta\left(K_{\mathrm{th}}-K_{0}\right)}, \\
& \forall \boldsymbol{\Gamma} \in D_{\alpha},
\end{aligned}
$$

where $K_{0}=(3 N-4) \beta^{-1} / 2$ is the fixed value of the kinetic energy of the thermostatting subsystem, has zero dissipation everywhere in the phase space domain $D_{\alpha}$. This distribution is therefore a time independent stationary distribution.

Let us assume that at time zero the initial distribution for the fully aged glass is not the quasiequilibrium distribution but instead is given by

$$
f_{\alpha}^{g}(\boldsymbol{\Gamma}, 0)=\frac{\exp \left[-\beta H_{0}(\boldsymbol{\Gamma})-g(\boldsymbol{\Gamma})\right] \delta(\mathbf{p}) \delta\left(K_{\mathrm{th}}-K_{0}\right)}{\int_{D_{\alpha}} d \boldsymbol{\Gamma} \exp \left[-\beta H_{0}(\boldsymbol{\Gamma})-g(\boldsymbol{\Gamma})\right] \delta(\mathbf{p}) \delta\left(K_{\mathrm{th}}-K_{0}\right)},
$$

$$
\forall \boldsymbol{\Gamma} \in D_{\alpha},
$$

where $g(\boldsymbol{\Gamma})$ is an arbitrary real valued deviation function (even in the momenta). Following Ref. 20, it is easy to see that the dissipation function is

$$
\Omega(\boldsymbol{\Gamma})=\dot{\boldsymbol{\Gamma}} \cdot \frac{\partial g}{\partial \boldsymbol{\Gamma}} \equiv \dot{g}(\boldsymbol{\Gamma}) .
$$

Substituting this expression for the dissipation into Eq. (6) gives

$$
f_{\alpha}^{g}(\boldsymbol{\Gamma}(0), t)=\exp [-\Delta g(\boldsymbol{\Gamma}(-t))] f_{\alpha}^{g}(\boldsymbol{\Gamma}(0), 0) .
$$

Now we assume that within the subdomain the system is $T$-mixing (transient mixing) as well as robust. This means that at long times $t>\tau$, transient time correlation functions of smooth zero mean variables factorize $\lim \langle a(0) b(s)\rangle$ $=\langle a(0)\rangle \lim \langle b(s)\rangle=0$. This implies that the only constants of $s \rightarrow \infty$

the motion are the thermostat momentum and kinetic energy as well as the subdomain occupation number $s_{\alpha}(\boldsymbol{\Gamma})$ $=0, \boldsymbol{\Gamma} \notin D_{\alpha}$ and $s_{\alpha}(\boldsymbol{\Gamma})=1, \boldsymbol{\Gamma} \in D_{\alpha}$ which basically says that the sample resides inside a robust subdomain of phase space $D_{\alpha}$. This implies that if there is any deviation from the quasiequilibrium distribution there will be dissipation and by the Second Law Inequality, the time average of the ensemble averaged dissipation (for a given sample glass) will be positive

$$
\left\langle\bar{g}_{t}\right\rangle_{\alpha}^{g}=\frac{1}{t}\langle g(t)-g(0)\rangle_{\alpha}^{g} \geq 0, \forall t .
$$

This equation is proved starting from the equations of motion in Ref. 20.

One may wonder about whether it is possible to have two separate regions in phase space: one with positive average dissipation and the second region with negative average dissipation such that the total average dissipation is zero. The Second Law Inequality proves that this situation is impossible. Consider a deviation function which is only nonzero for the region of supposed negative dissipation. If we consider the Second Law Inequality for this system, the Second Law Inequality says it has a positive average dissipation. So our assumption that there could be a region in phase space where the average dissipation is negative is, in fact, impossible. This proves that over the ergodic subdomain $D_{\alpha}$, the dissipationless distribution is unique. The average dissipation can only be zero if the distribution is the unique quasiequilibrium distribution in the ergodic subdomain of phase space $D_{\alpha}$.

If we now use the dissipation theorem ${ }^{26,27}$ to compute the ensemble averaged, time dependent dissipation where at time zero we start from an arbitrary distribution (even in momenta) Eq. (8) over the domain $D_{\alpha}$, then

$$
\langle g(t)\rangle_{\alpha}^{g}-\langle g(0)\rangle_{\alpha}^{g}=\int_{0}^{t} d s\langle\dot{g}(0) g(s)\rangle_{\alpha}^{g} .
$$

Now since the system is $T$-mixing over $D_{\alpha}$, at long times $t>\tau$, the transient time correlation function of zero mean variables factorizes $\lim _{s \rightarrow \infty}\langle\dot{g}(0) g(s)\rangle_{\alpha}^{g}=\langle\dot{g}(0)\rangle_{\alpha}^{g} \lim \langle g(s)\rangle_{\alpha}^{g}=0$ since $\langle\dot{g}(0)\rangle_{\alpha}^{g}=0$. At these long times, the transient time correlation function appearing in Eq. (12) goes to zero and the dissipation function does not change $\langle g(t)\rangle_{\alpha}^{g}=\langle g(\tau)\rangle_{\alpha}^{g}, \forall t$ $>\tau$ (i.e., at sufficiently long times there is no dissipation). This can only mean that the distribution has relaxed to the unique quasiequilibrium canonical distribution Eq. (7).

In fact, if the Deborah number is infinite and if at $t=0$, we perturb the phase space distribution of a fully aged glass, the phase space distribution must, at long times, return to a time independent distribution. The proof is simple: if this did not happen, the Deborah number of the initial sample could not possibly be infinite. The dissipation theorem shows that the transient time correlation function appearing in Eq. (12) must go to zero at long times, otherwise the system would never become time independent. Thus the fact that the Deborah number is effectively infinite implies the system is $T$-mixing.

Of course there may be very slow relaxation processes taking place in a glass. Eq. (10) shows that the slower the changes, the smaller is the dissipation function and the closer the actual distribution is to the quasiequilibrium distribution (7). The quasiequilibrium distribution can be approached arbitrarily closely by allowing the glass to age for a longer time. Eventually, the Deborah number becomes effectively infinite and correspondingly, the distribution function effectively becomes the quasiequilibrium distribution over the sample's ergodic subdomain. If the sample is $T$-mixing it must at long times relax to the unique dissipationless equilibrium distribution in some nonunique ergodic subdomain of phase space. The relaxation to quasiequilibrium need not be monotonic. This is because the transient time correlation function appearing in Eq. (12) is not necessarily positive for all times $s$.

It may seem odd that in very slowly relaxing glassy systems the system can still exhibit the $T$-mixing property. However, glasses are no different to crystalline solids in this respect. The order parameter for a crystal (which is the ana$\log$ of the subdomain occupation number for a glass) is static or robust. At long times, correlation functions of other zero mean variables decay to zero and crystalline solids at temperatures that are not too low exhibit the $T$-mixing property.

The derivation of the quasiequilibrium distribution func- 
tion for relaxed glassy systems given above complements the arguments given in Ref. 21 for the same distribution. In that paper, a series of gedanken experiments were used to construct the proof.

Of course, for typical glasses, there are an exceedingly large number of possible phase space domains that a sample may be arrested within and, almost certainly, our sample preparation protocols do not supply sufficient control to ensure that an ensemble of samples will all, after preparation, reside within a unique phase space domain.

If we consider the distribution function over a single domain $D_{\alpha}$, we define the subdomain partition function as

$$
Z_{\alpha}=\int_{D_{\alpha}} d \boldsymbol{\Gamma} \exp \left[-\beta H_{0}(\boldsymbol{\Gamma})\right] .
$$

When we consider the entire ensemble there are $N_{D}$ domains with the proportion of ensemble members in each domain being given by the weight $w_{\alpha}$ which is constrained such that $\sum_{\alpha=1}^{N_{D}} w_{\alpha}=1$. The distribution function for the entire ensemble is

$$
f_{q e}(\boldsymbol{\Gamma})=\sum_{\alpha=1}^{N_{D}} s_{\alpha}(\boldsymbol{\Gamma}) w_{\alpha} f_{q e, \alpha}(\boldsymbol{\Gamma})
$$

where the switch function is given as $s_{\alpha}(\boldsymbol{\Gamma})=1$ if $\boldsymbol{\Gamma} \in D_{\alpha}$ and $s_{\alpha}=0$ otherwise. We will refer to systems which are described by Eq. (14) with $N_{D} \geq 2$ as being in quasiequilibrium. We may obtain a quasiequilibrium ensemble average for some phase variable $B$ as

$$
\langle B\rangle_{q e}=\int d \boldsymbol{\Gamma} f_{q e}(\boldsymbol{\Gamma}) B(\boldsymbol{\Gamma}) .
$$

For many systems, we expect that as the system size is increased, the relative difference between a quasiequilibrium ensemble average of a smooth phase function obtained from Eq. (15) and a time average of the same quantity obtained from a single ensemble member decreases, and is insignificant in the thermodynamic limit.

\section{The shear modulus of glass}

In Ref. 19, we calculated (to leading order) the change in the $x y$ element of the pressure tensor for an equilibrium solid subject to a shearing deformation with a change in strain $\delta \gamma$ which provided an expression for the elastic modulus. The elastic modulus was required input for the Green-Kubo type theory we developed for the solid phase. To obtain the modulus, we perturbed the boundary conditions leading to an equivalent expression to that previously obtained by perturbing lattice sites, ${ }^{28,29}$ although our equivalent result was expressed in a different mathematical form.

Let us now consider what happens to a quasiequilibrium solid described by the quasiequilibrium distribution function Eq. (14) upon the application of a small strain. We assume that the phase space domains simply shear under the shearing deformation and thus in the comoving domains, the weights $w_{\alpha}$ do not change. This assumption will be valid if the domains are robust as specified in Ref. 21. The formalism for calculating such averages from quasiequilibrium fluctuation formula is specified in Refs. 21 and 30, (in particular, pedagogical examples for the heat capacity are presented).

To compute the response, we consider the effect of perturbing our initial domain, now denoted $D_{\alpha}(0)$, by having it flow with the perturbing strain and matching perturbation to the boundary conditions such that it becomes $D_{\alpha}(\delta \gamma)$. We generalize the switch function such that $s_{\alpha}(\boldsymbol{\Gamma}, \delta \gamma)=1$ if $\boldsymbol{\Gamma} \in D_{\alpha}(\delta \gamma)$ and $s_{\alpha}(\boldsymbol{\Gamma}, \delta \gamma)=0$ otherwise. The quasiequilibrium average for some phase variable $B$ is now given by the expression

$$
\langle B\rangle_{q e, \delta \gamma}=\int d \boldsymbol{\Gamma} f_{q e}(\boldsymbol{\Gamma}, \delta \gamma) B(\boldsymbol{\Gamma}),
$$

where

$$
f_{q e}(\boldsymbol{\Gamma}, \delta \gamma)=\sum_{\alpha=1}^{N_{D}} s_{\alpha}(\boldsymbol{\Gamma}, \delta \gamma) w_{\alpha} f_{q e, \alpha}(\boldsymbol{\Gamma}, \delta \gamma)
$$

and

$$
f_{q e, \alpha}(\boldsymbol{\Gamma}, \delta \gamma)=\frac{\exp \left[-\beta H_{0}(\boldsymbol{\Gamma})\right]}{Z_{\alpha}(\delta \gamma)},
$$

where

$$
Z_{\alpha}(\delta \gamma)=\int_{D_{\alpha}(\delta \gamma)} d \boldsymbol{\Gamma} \exp \left[-\beta H_{0}(\boldsymbol{\Gamma})\right]
$$

We wish to know the quasiequilibrium average of the $x y$ element of the pressure tensor $P_{x y}$ which is given by

$$
\left\langle P_{x y}\right\rangle_{q e, \delta \gamma}=\int d \boldsymbol{\Gamma} f_{q e}(\boldsymbol{\Gamma}, \delta \gamma) P_{x y}(\boldsymbol{\Gamma}) .
$$

To proceed, we use Eqs. (17)-(20) to obtain

$$
\left\langle P_{x y}\right\rangle_{q e, \delta \gamma}=\sum_{\alpha=1}^{N_{D}} w_{\alpha} \frac{\int_{D_{\alpha}(\delta \gamma)} d \boldsymbol{\Gamma} P_{x y}(\boldsymbol{\Gamma}) \exp \left[-\beta\left(H_{0}(\boldsymbol{\Gamma})\right]\right.}{\int_{D_{\alpha}(\delta \gamma)} d \boldsymbol{\Gamma} \exp \left[-\beta\left(H_{0}(\boldsymbol{\Gamma})\right]\right.} .
$$

Now $D_{\alpha}(\delta \gamma)$ defines a phase space domain which is strained an amount $\delta \gamma$ from a reference domain $D_{\alpha}(0)$. The transformation between the two domains is given by the equation

$$
\Gamma^{\prime} \equiv \boldsymbol{\Gamma}-\delta \Gamma
$$

where $\delta \boldsymbol{\Gamma}$ is given by

$$
\delta \boldsymbol{\Gamma}=\delta \gamma\left(q_{y 1}, 0,0, q_{y 2}, 0,0, \ldots, q_{y N}, 0,0,0, \ldots, 0,0\right)
$$

We can transform the average Eq. (21) using the coordinate transformation Eq. (22) to obtain 


$$
\left\langle P_{x y}\right\rangle_{q e, \delta \gamma}=\sum_{\alpha=1}^{N_{D}} w_{\alpha} \frac{\int_{D_{\alpha}(0)} d \boldsymbol{\Gamma}^{\prime}\left|\frac{\partial \boldsymbol{\Gamma}}{\partial \boldsymbol{\Gamma}^{\prime}}\right| P_{x y}\left(\boldsymbol{\Gamma}^{\prime}+\delta \boldsymbol{\Gamma}\right) \exp \left[-\beta\left(H_{0}\left(\boldsymbol{\Gamma}^{\prime}+\delta \boldsymbol{\Gamma}\right)\right]\right.}{\int_{D_{\alpha}(0)} d \boldsymbol{\Gamma}^{\prime}\left|\frac{\partial \boldsymbol{\Gamma}}{\partial \boldsymbol{\Gamma}^{\prime}}\right| \exp \left[-\beta\left(H_{0}\left(\boldsymbol{\Gamma}^{\prime}+\delta \boldsymbol{\Gamma}\right)\right]\right.} .
$$

Using the fact that the Jacobian is unity $\left|\partial \boldsymbol{\Gamma} / \partial \boldsymbol{\Gamma}^{\prime}\right|=1$, we can follow the steps in Ref. 30 to obtain

$$
\begin{aligned}
\left\langle P_{x y}\right\rangle_{q e, \delta \gamma}= & \sum_{\alpha=1}^{N_{D}} w_{\alpha}\left[\left\langle P_{x y}(\boldsymbol{\Gamma})\right\rangle_{\alpha, 0}+\left\langle\delta \boldsymbol{\Gamma} \cdot \nabla P_{x y}(\boldsymbol{\Gamma})\right\rangle_{\alpha, 0}\right. \\
& \left.-\beta\left\langle P_{x y}(\boldsymbol{\Gamma}) \delta \boldsymbol{\Gamma} \cdot \nabla H\right\rangle_{\alpha, 0}\right]\left[1+\beta\left\langle\delta \boldsymbol{\Gamma} \cdot \nabla H_{0}(\boldsymbol{\Gamma})\right\rangle_{\alpha, 0}\right] .
\end{aligned}
$$

where we use the notation

$$
\langle B\rangle_{\alpha, \delta \gamma}=\int_{D_{\alpha}(\delta \gamma)} d \boldsymbol{\Gamma} B(\boldsymbol{\Gamma}) f_{q e, \alpha}(\boldsymbol{\Gamma}, \delta \gamma)
$$

for the average over the $\alpha$ th domain. The $x y$ element of the pressure tensor $P_{x y}$ is given by

$$
V P_{x y}(\boldsymbol{\Gamma})=\sum_{i=1}^{N} \frac{p_{x i} p_{y i}}{m_{i}}+\sum_{i=1}^{N} F_{x i} q_{y i} .
$$

Applying the coordinate transformation Eq. (23), we see that

$$
\delta \boldsymbol{\Gamma} \cdot \nabla H_{0}(\boldsymbol{\Gamma})=-\delta \gamma P_{x y}^{\Phi}(\boldsymbol{\Gamma}) V,
$$

where $P_{x y}^{\Phi}=\Sigma F_{x i} q_{y i} / V$ is the configurational component of the $x y$ element of the pressure tensor. Further

$$
\delta \boldsymbol{\Gamma} \cdot \nabla P_{x y}(\boldsymbol{\Gamma}) V=\delta \gamma \sum_{i=1}^{N} \sum_{j=1}^{N} \frac{\partial F_{x i}}{\partial q_{x j}} q_{y i} q_{y j} .
$$

For simplicity, we define the phase function $g_{\infty}$ by the equation

$$
g_{\infty} V \equiv \sum_{i=1}^{N} \frac{p_{y i}^{2}}{m_{i}}-\sum_{i=1}^{N} \sum_{j=1}^{N} \frac{\partial F_{x i}}{\partial q_{x j}} q_{y i} q_{y j} .
$$

We note that for fluids and solids, the infinite frequency shear modulus is given by the ensemble average of $g_{\infty}$. We substitute these into Eq. (25) and obtain

$$
\begin{aligned}
\left\langle P_{x y}\right\rangle_{q e, \delta \gamma}= & \left\langle P_{x y}\right\rangle_{q e, 0}+\beta V \delta \gamma \sum_{\alpha=1}^{N_{D}} w_{\alpha}\left[\left\langle P_{x y}(\boldsymbol{\Gamma}) P_{x y}^{\Phi}(\boldsymbol{\Gamma})\right\rangle_{\alpha, 0}\right. \\
& \left.-\left\langle P_{x y}(\boldsymbol{\Gamma})\right\rangle_{\alpha, 0}\left\langle P_{x y}^{\Phi}(\boldsymbol{\Gamma})\right\rangle_{\alpha, 0}\right]+\sum_{\alpha=1}^{N_{D}} w_{\alpha}\left\langle\delta \boldsymbol{\Gamma} \cdot \nabla P_{x y}\right\rangle_{\alpha, 0} .
\end{aligned}
$$

For glasses the differences between different phase space subdomains are purely configurational (the momenta are able to be integrated from $-\infty$ to $\infty$ ) and so we obtain

$$
\begin{aligned}
\left\langle P_{x y}\right\rangle_{q e, \delta \gamma}= & \left\langle P_{x y}\right\rangle_{q e, 0}+\beta V \delta \gamma \sum_{\alpha=1}^{N_{D}} w_{\alpha}\left[\left\langle P_{x y}^{2}(\boldsymbol{\Gamma})\right\rangle_{\alpha, 0}\right. \\
& \left.-\left\langle P_{x y}(\boldsymbol{\Gamma})\right\rangle_{\alpha, 0}^{2}\right] \\
& -\delta \gamma \sum_{\alpha=1}^{N_{D}}\left\langle g_{\infty}\right\rangle_{\alpha, 0} .
\end{aligned}
$$

This implies the zero frequency shear modulus is

$$
G_{0}=\left\langle g_{\infty}\right\rangle_{q e, 0}-\beta V \sum_{\alpha=1}^{N_{D}} w_{\alpha}\left[\left\langle P_{x y}^{2}(\boldsymbol{\Gamma})\right\rangle_{\alpha, 0}-\left\langle P_{x y}(\boldsymbol{\Gamma})\right\rangle_{\alpha, 0}^{2}\right] .
$$

This generalizes, for the case of quasiequilibrium, the derivation of the correct finite temperature result first given by Squire et al. $^{28}$ in 1969 for equilibrium crystalline materials. For a fluid, the sum of these two terms is exactly zero since the shear modulus is zero, ${ }^{31}$ i.e.,

$$
\left\langle g_{\infty}\right\rangle_{q e, 0}^{\text {fluid }}=\beta V \sum_{\alpha=1}^{N_{D}} w_{\alpha}\left[\left\langle P_{x y}^{2}(\boldsymbol{\Gamma})\right\rangle_{\alpha, 0}-\left\langle P_{x y}(\boldsymbol{\Gamma})\right\rangle_{\alpha, 0}^{2}\right] .
$$

For a solid, these two terms do not cancel and there is a nonzero shear modulus.

An aspect of the quasiequilibrium distribution that is somewhat subtle is the following. Although the intradomain weights are Boltzmann because of the flexibility of the domains topology, the stress averaged over the quasiequilibrium distribution for an individual glass sample is nonzero in general. If the domain topology is strained a small amount, one can see that the average shear stress with a Boltzmann weight over domain $D_{\alpha}(\delta \gamma)$ is equal to a particular nonBoltzmann weighted average over domain $D_{\alpha}(0)$; this is made clear when we examine Eq. (31). The domain topology permits anisotropic averages even though the weight is formally isotropic. This is why a glass sample in general has residual stress. The symmetry of averages is a functional of both the formal Boltzmann weight and the domain topology.

\section{Linear response of glass to time dependent strain}

The standard derivations of linear response theory assume that the underlying equilibrium distribution function does not change with strain. Clearly, if we wish to treat a solid phase which is initially in either equilibrium or quasiequilibrium, we need to extend linear response theory by allowing the same strain rate to cause both a change to the underlying equilibrium state and nonequilibrium dissipation. 
Considering nonequilibrium perturbations to the quasiequilibrium distribution function Eq. (14) and the quasiequilibrium change to the strain given by Eq. (33).

Assuming domain robustness, and that the domains $D_{\alpha}(\gamma)$ stream with the perturbation denoted by $\gamma$ [given by the transformation Eq. (23)], we transform the domains $D_{\alpha}(\delta \gamma)$ with the strain transformation. Then the switches will be independent of the strain, i.e.,

$$
\left.s_{\alpha}\left(\boldsymbol{\Gamma}^{\prime}, 0\right)=s_{\alpha}(\boldsymbol{\Gamma}, \delta \gamma)\right) \forall \alpha, \boldsymbol{\Gamma}, \delta \gamma .
$$

[Remember from Eq. (22) that it is $\boldsymbol{\Gamma}$ that is the strained vector and $\boldsymbol{\Gamma}^{\prime}$ that is unstrained.] This will affect the normalization for the probability functions such that Eq. (13) depends on the perturbation, and thus we have Eqs. (17) and (18).

If the weights are independent of the strain

$$
w_{\alpha}\left(\boldsymbol{\Gamma}^{\prime}, 0\right)=w_{\alpha}(\boldsymbol{\Gamma}(\delta \gamma), \delta \gamma) \forall \alpha,
$$

we say that the domains are robust with respect to the strain. Certainly, this condition will not always hold. If we are too close to the glass transition, we have shown with a simulation study of the heat capacity that the domains cannot be expected to be robust with respect to small but finite changes in temperature or pressure. Indeed, in Ref. 21, we developed an independent test for the domains to be robust with respect to such changes. However, if the Deborah number is effectively infinite and if the response to a small strain scales linearly with the magnitude of the strain, the domains must be robust.

Further, as the transformation, Eqs. (22) and (23), has a unit Jacobian, given $A_{\alpha}(\delta \gamma)=-k_{B} T \ln \left(Z_{\alpha}(\delta \gamma)\right)$, following the analysis in Ref. 21, we have

$$
\begin{aligned}
\left.\frac{\partial A_{\alpha}(\delta \gamma)}{\partial \delta \gamma}\right|_{\delta \gamma=0} & =-\left.k_{B} T \frac{\partial}{\partial \delta \gamma} \ln \left[\int_{D_{\alpha}(\delta \gamma)} d \boldsymbol{\Gamma} \exp [-\beta H(\boldsymbol{\Gamma})]\right]\right|_{\delta \gamma=0} \\
& =-k_{B} T \frac{\int_{D_{\alpha}(0)} d \boldsymbol{\Gamma} \exp \left[-\beta H(\boldsymbol{\Gamma})-\beta \delta \boldsymbol{\Gamma} \cdot \nabla H_{0}(\boldsymbol{\Gamma})\right]-\int_{D_{\alpha}(0)} d \boldsymbol{\Gamma} \exp [-\beta H(\boldsymbol{\Gamma})]}{\delta \gamma \int_{D_{\alpha}(0)} d \boldsymbol{\Gamma} \exp [-\beta H(\boldsymbol{\Gamma})]}
\end{aligned}
$$

So using Eq. (27) to linear order, we have

$$
\begin{aligned}
\left.\frac{\partial A_{\alpha}(\delta \gamma)}{\partial \delta \gamma}\right|_{\delta \gamma=0} & =-\frac{\delta \gamma \int_{D_{\alpha}(0)} d \boldsymbol{\Gamma} P_{x y}^{\Phi}(\boldsymbol{\Gamma}) V \exp (-\beta H(\boldsymbol{\Gamma}))}{\delta \gamma \int_{D_{\alpha}(0)} d \boldsymbol{\Gamma} \exp [-\beta H(\boldsymbol{\Gamma})]} \\
& =-V\left\langle P_{x y}^{\Phi}\right\rangle_{\alpha, 0}=-V\left\langle P_{x y}\right\rangle_{\alpha, 0} .
\end{aligned}
$$

In general, for the equations of motion, Eq. (1), the phase space compression factor will be given in terms of the rate of change in energy due to the thermostatting term ${ }^{23,32}$ by $k_{B} T \Lambda(\boldsymbol{\Gamma})=-\dot{Q}(\boldsymbol{\Gamma})$ and the evolution of the probability distribution is given by the streaming version of the Liouville equation

$$
\frac{d}{d t} f(\boldsymbol{\Gamma}, t)=-f(\boldsymbol{\Gamma}, t) \Lambda(\boldsymbol{\Gamma}) .
$$

The formal solution of this equation is given by

$$
f(\boldsymbol{\Gamma}(t), t)=f(\boldsymbol{\Gamma}(0), 0) \exp \left(-\beta \int_{0}^{t} d s \dot{Q}(\boldsymbol{\Gamma}(s))\right),
$$

which may easily be verified by substitution into Eq. (39).

We thus define the time dependent distribution function for the ensemble members of an initially quasiequilibrium domain

$$
f_{n e, \alpha}(\boldsymbol{\Gamma}(t), t) \equiv f_{q e, \alpha}(\boldsymbol{\Gamma}(0), \gamma(0)) \exp \left(-\beta \int_{0}^{t} d s \dot{Q}(\boldsymbol{\Gamma}(s))\right),
$$

where from Eqs. (14), (40), and (41) we have

$$
f(\boldsymbol{\Gamma}(t), t)=\sum_{\alpha=1}^{N_{D}} s_{\alpha}(\boldsymbol{\Gamma}(0), \delta \gamma(0)) w_{\alpha} f_{n e, \alpha}(\boldsymbol{\Gamma}(t), t) .
$$

The microscopic analogue of the first law of thermodynamics may be expressed as

$$
\dot{H}_{0}(\boldsymbol{\Gamma})=-J(\boldsymbol{\Gamma}) V F_{e}(t)+\dot{Q}(\boldsymbol{\Gamma}),
$$

which defines the flux $J$ in terms of the work done by the external field $F_{e}$ and the heat absorbed by the system from the thermostat (usually negative). Detailed microscopic expressions for both quantities can be determined from the equations of motion, Eq. (1). For the case of planar shear $F_{e}=\dot{\gamma}(t)$ and $J=P_{x y}(\boldsymbol{\Gamma}) .{ }^{23}$ Using Eqs. (18), (41), and (43) under the robustness condition for the strained domains during the time interval $0 \leq s \leq t$, it is easy to show, for a system initially in quasiequilibrium at time $s=0$, that

$$
\begin{aligned}
f_{n e, \alpha}(\boldsymbol{\Gamma}(t), t)= & f_{q e, \alpha}(\boldsymbol{\Gamma}(t), \delta \gamma(t)) \exp \left[-\beta \int_{0}^{t} d s\left(J(\boldsymbol{\Gamma}(s)) V F_{e}(s)\right.\right. \\
& \left.\left.+\dot{\gamma}(s) \frac{d A_{\alpha}(\delta \gamma(s))}{d \delta \gamma}\right)\right]
\end{aligned}
$$

where we have made use of the fact that

$$
\exp \left(-\beta \int_{0}^{t} d s \dot{\gamma}(s) \frac{\partial A_{\alpha}(\delta \gamma(s))}{\partial \delta \gamma}\right)=\frac{Z_{\alpha}(\delta \gamma(t))}{Z_{\alpha}(\delta \gamma(0))} .
$$

Following the transformation procedure in Ref. 33, we define the initial point as $\boldsymbol{\Gamma} \equiv \boldsymbol{\Gamma}(0)$ and introduce the substitution for the phase variable $\boldsymbol{\Gamma}^{\dagger}=\boldsymbol{\Gamma}(t)$, then $\boldsymbol{\Gamma}^{\dagger}(s) \equiv \boldsymbol{\Gamma}(t+s)$, and $\boldsymbol{\Gamma}(s)$ 
$=\boldsymbol{\Gamma}^{\dagger}(s-t)$. We now substitute $\boldsymbol{\Gamma}^{\dagger}$ into Eq. (44) and noting that $\boldsymbol{\Gamma}^{\dagger}$ is merely a dummy variable we drop the dagger $\dagger$ to obtain

$$
\begin{aligned}
f_{n e, \alpha}(\boldsymbol{\Gamma}, t)= & f_{q e, \alpha}(\boldsymbol{\Gamma}, \delta \gamma(t)) \exp \left[-\beta \int_{0}^{t} d s\left(J(\boldsymbol{\Gamma}(s-t)) V F_{e}(s)\right.\right. \\
& \left.\left.+\dot{\gamma}(s) \frac{d A_{\alpha}(\delta \gamma(s))}{d \delta \gamma}\right)\right]
\end{aligned}
$$

For Eq. (42) this transformation results in

$$
f(\boldsymbol{\Gamma}, t)=\sum_{\alpha=1}^{N_{D}} s_{\alpha}(\boldsymbol{\Gamma}(-t), \delta \gamma(0)) w_{\alpha} f_{n e, \alpha}(\boldsymbol{\Gamma}, t) .
$$

We now make the substitution $v=t-s$ and upon replacing the dummy variable $v$ with $s$ we obtain

$$
\begin{aligned}
f_{n e, \alpha}(\boldsymbol{\Gamma}, t)= & f_{q e, \alpha}(\boldsymbol{\Gamma}, \delta \gamma(t)) \exp \left[-\beta \int_{0}^{t} d s\left(J(\boldsymbol{\Gamma}(-s)) V F_{e}(t-s)\right.\right. \\
& \left.\left.+\dot{\gamma}(t-s) \frac{d A_{\alpha}(\delta \gamma(t-s))}{d \delta \gamma}\right)\right] .
\end{aligned}
$$

which properly separates the phase variable $\boldsymbol{\Gamma}$ from the temporal parameter $t$. In Eqs. (47) and (48), $\boldsymbol{\Gamma}(-s)$ is the phase space vector we arrive at if we start at $\boldsymbol{\Gamma}$ and run the equations of motion back in time for the duration $s$ using the protocol $\boldsymbol{F}_{e}(t-u), \gamma(t-u)$, initially with the time variable $u$ $=0$ and finally with $u=s$. Combining Eqs. (47) and (48) we obtain,

$$
\begin{aligned}
f(\boldsymbol{\Gamma}, t)= & \sum_{\alpha=1}^{N_{D}} s_{\alpha}(\boldsymbol{\Gamma}(-t), \delta \gamma(0)) w_{\alpha} f_{q e, \alpha}(\boldsymbol{\Gamma}, \delta \gamma(t)) \\
& \times \exp \left[-\beta \int_{0}^{t} d s\left(J(\boldsymbol{\Gamma}(-s)) V F_{e}(t-s)\right.\right. \\
& \left.\left.+\dot{\gamma}(t-s) \frac{d A_{\alpha}(\delta \gamma(t-s))}{d \delta \gamma}\right)\right] .
\end{aligned}
$$

To proceed, we assume that

$$
s_{\alpha}(\boldsymbol{\Gamma}(t), \delta \gamma(t))=s_{\alpha}(\boldsymbol{\Gamma}(t+\tau), \delta \gamma(t+\tau)) \forall \alpha, t, \tau,
$$

i.e., on the time scales we consider, the number of transitions between different domains is insignificant and the Deborah number is effectively infinite. We now consider the leading order change to an average, by taking the first order Taylor expansion of the exponential in Eq. (49) to obtain the desired linear response theory

$$
\begin{aligned}
\langle B(t)\rangle= & \langle B\rangle_{q e, \delta \gamma(t)}-\beta V \int_{0}^{t} d s F_{e}(t-s) \\
& \times \sum_{\alpha=1}^{N_{D}} w_{\alpha}\langle J(\boldsymbol{\Gamma}(-s)) B(\boldsymbol{\Gamma})\rangle_{\alpha, 0} \\
& -\beta \int_{0}^{t} d s \dot{\gamma}(t-s) \sum_{\alpha=1}^{N_{D}} w_{\alpha}\langle B(\boldsymbol{\Gamma})\rangle_{\alpha, 0} \frac{d}{d \delta \gamma} A_{\alpha}(\delta \gamma=0),
\end{aligned}
$$

where all terms of $\mathcal{O}\left(F_{e}^{2}\right), \mathcal{O}\left(\delta \gamma^{2}\right)$, or higher have been ignored. If we substitute the variables $\lambda, \dot{\lambda}, \delta \lambda$ for $\gamma, \dot{\gamma}, \delta \gamma$ in Eq. (51) we may use this response theory for other processes.

We need to now comment on the use of these formulae in the context of the glass transition. When we are dealing with a fluid, even a supercooled liquid, we cannot use the results above. There is no change in the Helmholtz free energy with strain. As we lower the temperature and cross the glass transition, when viewed as a fluid, the material properties are continuous functions of temperature. As the shear viscosity becomes ever greater, it becomes so large that we cannot even measure the zero frequency shear viscosity any more. So when we decide that the Deborah number is effectively infinite, we have to use the formulae given above. The formulae for material properties change. Thus there is a discontinuous change in the material properties because of the discontinuous change in the relations between microscopic fluctuations and averages, and macroscopic material properties: we are dealing with a solid. As we said in Sec. I, the choice of the precise transition of formulae for solids to using formulae for fluids is, of course, subjective. In the transition region itself the domains are not robust and no presently known formalism can be applied.

\section{E. Oscillatory planar shear}

We now consider the case of oscillatory planar shear applied to a quasiequilibrium system, by using Eq. (51) to calculate the response in the stress $B(\boldsymbol{\Gamma})=J(\boldsymbol{\Gamma})=P_{x y}(\boldsymbol{\Gamma})$ to an applied strain of the form

$$
\gamma(t)=\gamma_{0} \sin (\omega t)=-\mathfrak{R}\left[i \gamma_{0} e^{i \omega t}\right] .
$$

The response to the oscillatory strain will become sinusoidal after the decay of initial transients and is often expressed in terms of the storage $\widetilde{G}_{R}$ and loss $\widetilde{G}_{I}$ shear moduli

$$
\lim _{t \rightarrow \infty}\left\langle P_{x y}(t)\right\rangle=\mathfrak{R}\left[i \gamma_{0} \widetilde{G} \exp (i \omega t)\right]
$$

where $\tilde{G}(\omega)=\widetilde{G}_{R}(\omega)+i \widetilde{G}_{I}(\omega)$. This quantity is related to the complex frequency dependent shear viscosity by the equation $\tilde{G}(\omega)=i \omega \tilde{\eta}(\omega)$. Thus, $G_{0}=\Re[\widetilde{G}(\omega=0)]$ and $G_{\infty}$ $=\lim \mathfrak{R}[\tilde{G}(\omega)]$.

$\omega \rightarrow \infty$

The applied field is $F_{e}=\dot{\gamma}(t)=\omega \gamma_{0} \cos (\omega t)$, the change in free energy for a single domain is given by $d A_{\alpha} / d \gamma$ $=-\left\langle P_{x y}\right\rangle_{e q, \alpha}$, and the flux is $J(\boldsymbol{\Gamma})=P_{x y}(\boldsymbol{\Gamma})$. So if we use Eq. (51) for this case, we obtain

$$
\left\langle P_{x y}(t)\right\rangle=\left\langle P_{x y}\right\rangle_{q e, \delta \gamma(t)}-\beta V \int_{0}^{t} d s \dot{\gamma}(t-s) \sum_{\alpha=1}^{N_{D}} w_{\alpha} C_{\alpha}(s),
$$

where

$$
C_{\alpha}(s)=\left\langle\Delta P_{x y}(\boldsymbol{\Gamma}(-s)) \Delta P_{x y}(\boldsymbol{\Gamma}(0))\right\rangle_{\alpha, 0},
$$

and where $\Delta P_{x y}(\boldsymbol{\Gamma})=P_{x y}(\boldsymbol{\Gamma})-\left\langle P_{x y}\right\rangle_{\alpha, 0}$. For a fluid the first term on the right hand side of Eq. (54) is zero and in Eq. (55) $\left\langle P_{x y}\right\rangle_{\alpha, 0}=\left\langle P_{x y}\right\rangle_{0}=0$.

We can rewrite Eq. (54) as 


$$
\begin{aligned}
\left\langle P_{x y}(t)\right\rangle_{q e, \delta \gamma}= & \left\langle P_{x y}\right\rangle_{q e, 0}-G_{0} \delta \gamma(t) \\
& -\beta V \int_{0}^{t} d s \dot{\gamma}(t-s) \sum_{\alpha=1}^{N_{D}} w_{\alpha} C_{\alpha}(s) .
\end{aligned}
$$

We can simplify this equation by introducing the ensemble averaged memory kernel

$$
\eta_{g}(t) \equiv \beta V \sum_{\alpha=1}^{N_{D}} w_{\alpha} C_{\alpha}(t)
$$

giving

$$
\left\langle P_{x y}(t)\right\rangle_{q e, \delta \gamma}=\left\langle P_{x y}\right\rangle_{q e, 0}-G_{0} \delta \gamma(t)-\int_{0}^{t} d s \dot{\gamma}(t-s) \eta_{g}(s) .
$$

For a fluid the first two terms on the right hand side of Eq. (58) are zero and we have a fluid time dependent linear response given by the well known equation

$$
\left\langle P_{x y}(t)\right\rangle_{f, \delta \gamma} \equiv-\int_{0}^{t} d s \dot{\gamma}(t-s) \eta_{f}(s),
$$

where the subscript " $f$ " denotes that the system is a fluid. Now if we try to view a solid glass as a (supercooled) fluid, we have to make the following transformation between fluid and glass memory functions

$$
\eta_{f}(t)=\eta_{g}(t)+G_{0},
$$

where $\eta_{g}(t)$ is defined by Eq. (57). For a solid glass, $\eta_{f}(t)$ is simply a memory kernel and unlike $\eta_{g}(t)$, is not defined in terms of stress autocorrelations. The shear modulus is given by Eq. (33). One also has of course to supplement Eq. (59) with the ensemble averaged residual stress. Viewing Eq. (60) as a memory kernel for a fluid, it is easy to see why if we view a solid glass as a fluid, the apparent zero frequency shear viscosity $\tilde{\eta}_{f}(0)=\int_{0}^{\infty} \eta_{f}(t) d t$ is infinite. On the other hand, at all nonzero frequencies the frequency dependent fluid viscosity is the same as the frequency dependent glass viscosity: $\widetilde{\eta}_{f}(\omega)=\widetilde{\eta}_{g}(\omega), \forall \omega \neq 0$. This is precisely why the subjective decision to classify a glass as a solid below the glass transition has a discontinuous effect on both material properties and on the connection between statistical mechanical quantities and material properties.

You can also see how the fluid viscosity diverges as the glass transition is approached from the high temperature side. The fluid memory kernel gains an increasingly long ranged and increasingly flat "molasses tail" as the glass transition is approached. Eventually the Deborah number becomes so large we decide to classify the material as a solid. At this stage three things happen. We regard the residual stress as nondecaying and we subtract the residual stress from the stress appearing in the time correlation functions Eq. (55). This removes the apparently diverging zero frequency shear viscosity. Secondly, we must add the zero frequency shear modulus to the presumed fluid memory kernel Eq. (60) or equivalently we add the shear modulus to Eq. (58). Thirdly, we must add the residual stress as the first term in Eq. (58).
It is worth noting that in Eq. (60) the history dependent zero frequency shear modulus is a quasiequilibrium property quite unrelated to Green-Kubo time correlation functions.

Using the trigonometric identity $\cos (\omega(t-s))$ $=\cos (\omega t) \cos (\omega s)+\sin (\omega t) \sin (\omega s)$, we obtain the following expressions for the storage and loss moduli:

$$
\begin{aligned}
& \widetilde{G}_{R}(\omega)=G_{0}+\beta V \omega \int_{0}^{\infty} d s \sin (\omega s) C_{q e}(s), \\
& \tilde{G}_{I}(\omega)=\beta V \omega \int_{0}^{\infty} d s \cos (\omega s) C_{q e}(s),
\end{aligned}
$$

where

$$
C_{q e}(t)=\sum_{\alpha=1}^{N_{D}} w_{\alpha} C_{\alpha}(t),
$$

and the zero frequency quasiequilibrium modulus $G_{0}$ is given by Eq. (33). Note that the functions $C_{\alpha}(t)$ are autocorrelation functions with the average squared subtracted off [see Eq. (55)].

\section{SIMULATION, RESULTS, AND DISCUSSION}

\section{A. Simulation details}

Our model glass former ${ }^{21,34}$ uses the following variation on the potential which was introduced by Kob and Andersen, ${ }^{35}$

$$
\begin{aligned}
& u_{i j}\left(r_{i j}\right)=4 \epsilon_{\alpha \beta}\left[\left(\frac{\sigma_{\alpha \beta}}{r_{i j}}\right)^{12}-\left(\frac{\sigma_{\alpha \beta}}{r_{i j}}\right)^{6}+\frac{1}{4}\right] \forall r_{i j}<2^{1 / 6} \sigma_{\alpha \beta}, \\
& u_{i j}\left(r_{i j}\right)=0 \forall r_{i j} \geq 2^{1 / 6} \sigma_{\alpha \beta},
\end{aligned}
$$

where the species identities of particles $i$ and $j$, either $A$ or $B$, are denoted by the subscripts $\alpha$ and $\beta$. The energy parameters are set to $\epsilon_{B B}=0.5 \epsilon_{A A}, \epsilon_{A B}=1.5 \epsilon_{A A}$, and the particle interaction distances $\sigma_{B B}=0.88 \sigma_{A A}, \sigma_{A B}=0.8 \sigma_{A A}$. The energy unit is $\epsilon_{A A}$, the length unit is $\sigma_{A A}$, and the time unit is $\sqrt{m \sigma_{A A}^{2} / \epsilon_{A A}}$ with both species having the same mass $m$. The composition is set to $X=N_{B} / N=0.2$, the number of particles is $N=N_{A}+N_{B}=108$, the density is $\rho=N / V=1.25$ for $T=0.6$ and $\rho=1.3$ for $T=0.3$. The density $\rho=1.25$ corresponds to the average density of the constant pressure simulations in Ref. 21 at the temperature of $T_{g}=0.435$ which was identified as the nominal glass transition temperature based on self diffusion data. Increasing the density increases the glass transition temperature. This means the simulations at $T=0.6$ are in a fluid state (possibly supercooled) and the simulations at $T$ $=0.3$ are in a nonequilibrium glass state. All simulations were repeated 1000 times.

To test the theory, we used both equilibrium time correlation data, obtained using Eq. (1) with $\mathbf{F}_{e}=0$ and nonequilibrium molecular dynamics data, obtained from Eq. (2) from oscillatory strain simulations. The amplitude of the strain was fixed at $\gamma_{0}=0.025$. This means that at low frequencies the signal to noise ratio deteriorates because the strain rate goes toward zero. 


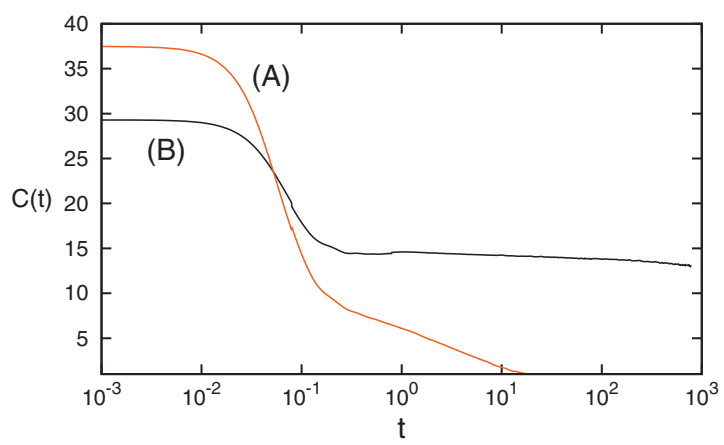

FIG. 1. The stress correlation functions for (a) the supercooled fluid at the temperature $T=0.6$, which is the curve with the initial value of $C(t) \simeq 37$ that fully decays, $C(t)=V\left\langle P_{x y}(t) P_{x y}(0)\right\rangle$ and (b) for the glass at the temperature $T=0.3$, which is the curve with the initial value of $C(t) \simeq 29, C(t)$ $=V \Sigma_{\alpha=1}^{N_{D}} w_{\alpha}\left\langle P_{x y}(t) P_{x y}(0)\right\rangle_{\alpha}$.

\section{B. Supercooled fluid phase}

We start by examining the case of a viscous supercooled fluid at the temperature of $T=0.6$. This is well above the nominal glass transition temperature of $T=0.435$. We calculated the correlation function $C(t)=V\left\langle P_{x y}(t) P_{x y}(0)\right\rangle$, which may be seen in Fig. 1. We fitted this to the functional forms given in the appendix and then Fourier transformed the resulting function, according to Eq. (61) with $N_{D}=1$ and $G_{0}$ $=0$, using the first order Filon's quadrature (see the Appendix of Ref. 19), and obtained the storage and loss moduli. We then performed nonequilibrium simulations at various frequencies, and applied a least squares fit of a sinusoidal function to the response allowing us to obtain estimates of $\widetilde{G}$ at that small number of frequencies. The results of this are shown in Fig. 2. It can be seen that the agreement between the two data sets is very good except for the data at the lowest frequency. In general, the low frequency data are very difficult to obtain reliably. As mentioned above, since the strain amplitude is fixed, the strain rate becomes very small at low frequencies with $\dot{\gamma} \sim \omega$. It can be seen that the low frequency data, from the transformed $C(t)$, decays as, $\lim _{\omega \rightarrow 0} \ln \left[\widetilde{G}_{R}(\omega)\right] \sim 2 \ln (\omega)+\ln \left[\beta V \int_{0}^{\infty} C(s) d s\right]$, (slope of 2 in the figure) and $\lim _{\omega \rightarrow 0} \ln \left[\widetilde{G}_{I}(\omega)\right]=\ln (\omega)+\ln (\eta)$ (slope of unity in the figure). This is obvious from Eq. (61), given that $G_{0}=0$ for the supercooled fluid, upon taking the small angle approximations, $\sin (\omega s) \simeq \omega s$ and $\cos (\omega s) \simeq 1$, we see that $\lim _{\omega \rightarrow 0} \widetilde{G}_{R}(\omega) \sim \beta V \omega^{2} \int_{0}^{\infty} d s s C(s)$ and $\lim _{\omega \rightarrow 0} \widetilde{G}_{I}(\omega)=\omega \eta$. So if this behavior is observed for both the storage and the loss modulus, on the same time scale, we have the signature of a fluid. From the data presented here we see that $\eta \simeq 200$. This is much greater than the corresponding crystal $(\eta \simeq 2.5)$ detailed in Ref. 19 (see erratum).

\section{Amorphous solid phase}

We now move on to the considerably more difficult case of the glass. We use the temperature of $T=0.3$ at the density of $\rho=1.3$ which puts us well below the nominal glass transition temperature. The average for the quantity $\Sigma_{\alpha=1}^{N_{D}} w_{\alpha}\left\langle P_{x y}\right\rangle_{e q, \alpha}^{2}$ which appears in Eq. (33) requires us to directly consider the phase space domains. This may be done by treating each simulation as ergodically sampling its own domain and then using time averaging. This approach is numerically difficult and the results can be sensitively dependent on the amount of time that is chosen to construct the averages over. We can avoid the need to compute it by noting [see Eq. (33)] that $G_{0, q e}=\left\langle g_{\infty}\right\rangle_{q e}-\beta V C_{q e}(0)$. We then compute the correlation function, $\left\langle P_{x y}(0) P_{x y}(t)\right\rangle_{q e}$, out to a cut off time $t_{c}=800$, and then assume that $C_{q e}(t)=\left\langle P_{x y}(0) P_{x y}(t)\right\rangle_{q e}$ $-\left\langle P_{x y}(0) P_{x y}\left(t_{c}\right)\right\rangle_{q e}$ for times up to $t_{c}$ and zero for all times beyond this. As we can compute the value of $G_{0, q e}$ without reference to $\left\langle P_{x y}\right\rangle_{q e, \alpha}$ and as the value of $d C_{q e}(t) / d t$ is already approaching zero at time $t=t_{c}$ (see Fig. 1) the properties of the Fourier transform leave this assumption irrelevant for frequencies $\omega \gg 1 / t_{c}$.

After the quench the simulations were subject to an oscillatory planar shear of amplitude $\gamma_{0}=0.15$ and period $10^{3}$ for a duration of $10^{4}$ and then left to age (for a duration of $\left.t_{\text {age }} \sim 1.6 \times 10^{4}\right)$ to the quasiequilibrium state. In terms of Lennard-Jones units for argon, this is $\sim 10 \mathrm{ps,}$, and so our system is very poorly aged compared to a macroscopic glass. Because of this very short time scale, the point we identify as the glass transition has a viscosity (in argon units) more like that of cold honey than a typical real glass with a viscosity of $10^{13}$ poise at the transition. If our theory is successful for such poorly aged simulations it should be very good for many real experimental systems.
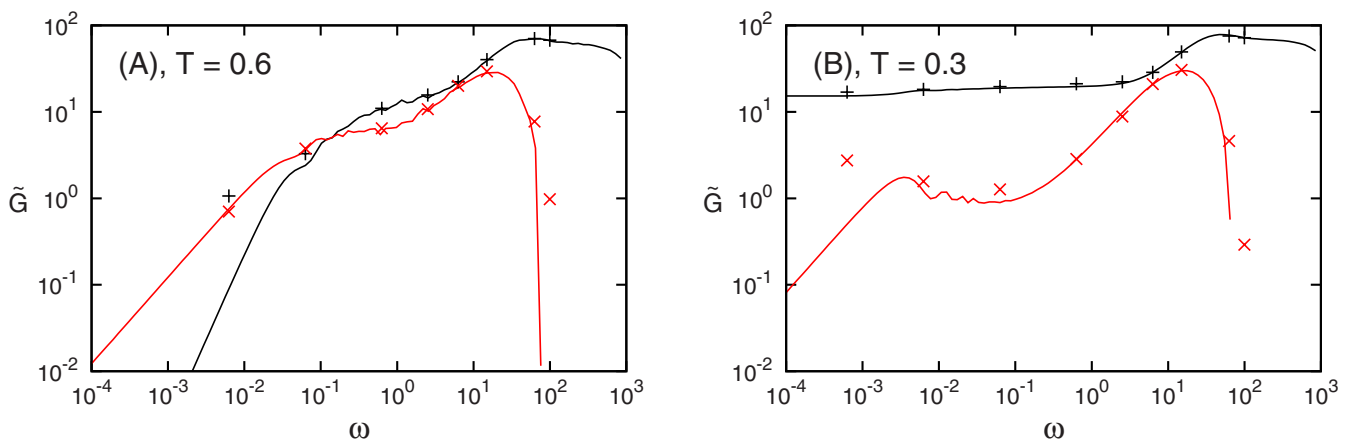

FIG. 2. The storage $\widetilde{G}_{R}$ and loss $\widetilde{G}_{I}$ moduli $\widetilde{G}=\widetilde{G}_{R}+\widetilde{G}_{I}$. The supercooled fluid at temperature $T=0.6$ is denoted (a) while the glass results at temperature $T$ $=0.3$ is denoted (b). The storage modulus $\widetilde{G}_{R}$ is the black curve and symbols $(+)$. The loss modulus $\widetilde{G}_{I}$ is the red curve with the symbols $(\times)$, which rapidly decays toward zero at high frequencies. The solid curves are obtained from linear response theory using data obtained from the (quasi-)equilibrium simulations while the symbols were obtained directly from the nonequilibrium molecular dynamics simulations. 
The nonequilibrium simulations as well as the quasiequilibrium simulations were computed. The results for the correlation function $\left\langle P_{x y}(t) P_{x y}(0)\right\rangle_{q e}$ may be seen in Fig. 1 and the results for the storage and loss moduli from both the response theory and the simulations may be seen in Fig. 2. Clearly the results compare very favorably for all frequencies with the exception of the lowest one, showing how our theory is very successful for even this relatively poorly aged glass. From the simulations we obtain $\widetilde{G}_{R}(\infty) \neq \beta V\left\langle P_{x y}^{2}\right\rangle_{q e}$ $\left(\widetilde{G}_{R}(\infty)=69 \pm 1\right.$ and $\left.\beta V\left\langle P_{x y}^{2}\right\rangle=97 \pm 1\right)$ showing that over the timescales that are accessible to us, the system is fundamentally solid in nature.

It is clear from the graphs that within the available frequency range, the zero frequency elastic constant for the supercooled fluid appears consistent with its value being zero. On the other hand the zero frequency shear modulus of the glass appears to be $\sim 20$.

We have already seen that the limiting zero frequency shear viscosity of the supercooled liquid is $\eta \sim 200$. Due to the limited time scale that we are able to access with the simulations and the limited amount of aging we can subject the glass to, we can say little about its limiting zero frequency viscosity. However, we can conclude that the viscosity for the glass, when treated as a very slow fluid, must be $\eta \gg 10^{3}$. The value for the supercooled liquid is some two orders of magnitude greater than that of the corresponding crystal $^{19}$ which has a similar viscosity to the fluid at the triple point. It is no surprise that the viscosity of the glass is so great. In fact, the glass transition is often defined as occurring at the temperature where the supercooled fluid reaches a viscosity of $10^{13}$ poise.

However, it is important to consider the implications of this in more detail. In our calculation we assume that the stress autocorrelation function has a tail that does not decay to zero. We treat the glass exactly as we did in a recent paper on the limiting zero frequency viscosity of a crystal. ${ }^{19}$ The shear stress contains two components. It is the sum of the residual stress that does not decay on any accessible timescale and the decaying stress. When it is said that a glass is a supercooled liquid with a viscosity greater than $10^{13}$ poise that shear viscosity is calculated from the time correlation function of the total stress, including the residual stress. Thus the viscosity diverges to infinity. Upon approaching the glass transition, it becomes difficult to obtain a history independent metastable fluid state and it also becomes hard to decide whether there is a nonzero value for the elastic modulus. These two problems arise from the same cause, namely, the slowness of all relaxation time scales. Of course, in computer simulations, the timescales are very limited compared to timescales available to laboratory experiments. Our "glassy" system may very well have a zero value for the elastic modulus, if we were able to simulate for a sufficiently long time to observe complete stress relaxation. This time would need to be long enough that the system reaches a history independent metastable fluid state, i.e., long enough to melt the glass. Nevertheless, our theory shows good agreement between nonequilibrium molecular dynamics computation of the frequency dependent moduli and the predictions of our time dependent response theory for the history dependent glass state. To do this, we have had to treat the glass as a solid, not a slow fluid.

\section{CONCLUSIONS}

As Max Born stated in 1939 “...there can be no ambiguity in the definition of, or the criterion for, melting. The difference between a solid and a liquid is that the solid has elastic resistance to shearing stress while a liquid does not." We have presented a statistical mechanical analysis of a supercooled liquid and a glass that shows that on accessible timescales, below the glass transition temperature, a glass has a nonzero value for the zero frequency shear modulus $G_{0}=\widetilde{G}_{R}(0)$, while above the glass transition that same modulus appears to vanish. We employed our recently developed quasiequilibrium phase space distribution for an ensemble of glass samples. Because individual glass samples are not in true thermodynamic equilibrium, the time averaged stress of an individual aged glass sample is nonzero in general. This is the residual stress of a glass sample. The quasiequilibrium distribution for a glass is history dependent and consists of a sum of nonoverlapping sample distributions. Within an individual ergodic subdomain, the weights are Boltzmann. The interdomain weights are history dependent and nonBoltzmann. In spite of this complexity, provided the subdomains are robust with respect to small changes in the relevant thermodynamic variables, we can derive correct statistical mechanical expressions for various thermodynamic quantities.

In this paper, we have concentrated on expressions for both static and frequency dependent elastic constants. The frequency dependent elastic constants and viscosities are derived from linear response theory adapted to the initial quasiequilibrium distribution function. Because we are only interested in the linear response, the phase space subdomains must be robust with respect to the linearly applied strains, at least over accessible timescales. In other words the subdomain $D(\delta \gamma)$, distortions, caused by the strain $\delta \gamma$, must be higher order than linear (e.g., $D(\delta \gamma)-D(0)$ $=O\left(\delta \gamma^{2}\right), O\left(\delta \gamma^{3}\right)$, etc. $)$ for the theory to work. As noted in our earlier work we could use the Evans-Searles transient fluctuation theorem to directly test this. In the present paper we have not done this. The fact that within experimental uncertainties the response theory results match the directly computed nonequilibrium ensemble averages indicates that for the states considered here, which are away from the glass transition itself, the domains are at least linearly robust with respect to strain deformations.

Our two key observational results, namely, that over timescales that are accessible to us, the zero frequency shear modulus of the glass is nonzero (i.e., $\widetilde{G}_{R}(\infty) \neq \beta V\left\langle P_{x y}^{2}\right\rangle_{\text {glass }}$ ), while the shear modulus of the supercooled liquid is zero, confirm that over accessible timescales, an aged glass should be viewed rheologically as a solid rather than a fluid. This means that we must use a different stress strain rate constitutive relation for a solid compared to a liquid. Also, the shear viscosity is determined by the integral of the autocorrelation function of the difference in the shear stress from the 
residual stress fluctuations. In changing from the GreenKubo integral associated with the fluid to that associated with the glass we induce a discontinuous change in the zero frequency shear modulus as well as the frequency dependent shear viscosity. However, in our calculations, it is very difficult to determine the shear modulus accurately, near the glass transition. The Green-Kubo integral for the glass is able to describe the behavior of the glass on the relevant time scale where it is in a quasiequilibrium state.

It is important to remember that in order to accurately be able to apply our formulae to compute the frequency dependent viscosities or elastic moduli near the glass transition, we not only require very long times for the correlation function integrals to converge, but even before we attempt to calculate equilibrium or quasiequilibrium time correlation function, we must wait for the samples to age. As the glass transition is approached this time also diverges to infinity. The aging process involves two processes. The slowest of these involves the aging of the topology of the phase space subdomains themselves. These subdomains need to become time independent and robust. Within these aged domains, the distributions then need enough time to relax to quasiequilibrium.

All states of matter, gas, liquid, supercooled liquid, glass and crystal, each have nonzero values for the limiting zero frequency shear viscosity. Shear viscosity therefore cannot be used as the fundamental diagnostic of the rheological character of these states of matter. Of these states of matter, the supercooled liquid has the highest limiting shear viscosity. Extrapolated to the glass transition it is in fact infinity [see Eq. (60)]. Not too far below the glass transition the (solid) glass has a much lower limiting viscosity than the supercooled liquid but still has a higher viscosity than the crystal or the liquid. Gases, of course, have the lowest shear viscosities. It is interesting to note the respective viscosity temperature coefficients are negative for the liquid (both supercooled and normal) but positive for the crystal, (solid) glass, and gas.

If the glass transition is approached from the high temperature side, then the supercooled liquid always has a zero value for the elastic modulus and the viscosity grows without bound as the glass transition is approached. This increase in viscosity lengthens all relaxation times especially the time required for aging and the time for the stress autocorrelation function to decay to zero. Eventually, the Deborah number becomes so large that we observe a history dependence, which does not relax on the longest available observation time. To accurately model this history dependent glassy state, we must treat it as an elastic solid with a nonzero elastic constant and residual stress. Viewed as a liquid, what became the elastic modulus contributed to the divergent viscosity Eq. (60). Viewed as a liquid, the residual stress which had been slowly decaying above the glass transition now also contributes to the divergent viscosity. Viewed as a solid, the residual stress and the elastic modulus both cease to contribute to the limiting zero frequency shear viscosity. This inevitably results in a discontinuous change in the low frequency shear viscosity at the glass transition.

The precise decision about when to say the glass is a solid is subjective. However the subjectivity of this transition is ameliorated somewhat due to the fact that as the glass transition is approached from above the shear viscosity and the relaxation times increases extremely rapidly. Our numerical results show that in this regime, our response theory can be used to self consistently model the rheological behavior of the history dependent solid glass at least over the frequency range that is accessible to us.

We have a few remarks regarding the quasiequilibrium phase space distribution. It may seem odd that in a nonequilibrium system such as a glass that we can make any definitive statements about phase space distributions. In our original derivation of the quasiequilibrium distribution, ${ }^{21}$ we used a gedanken experiment to prove that within a fully relaxed (time independent) ergodic subdomain the intradomain weights must be Boltzmann. In the present paper we have derived the same results using a completely different set of arguments based on the relaxation theorem. ${ }^{20}$ These results rest on the huge separation of timescales between interdomain relaxation and intradomain relaxation. This extremely large separation allows the intradomain distribution of states to be given by Boltzmann statistics while the interdomain distribution is highly nonequilibrium and static over experimentally accessible timescales. Indeed, that extreme slowness of domain relaxation must also imply domain robustness which is necessary for our theory to yield correct results. This large separation of timescales breaks down close to the actual glass transition and consequently the theory is not useful in that region. This breakdown in the immediate neighborhood of the glass transition also means that thermodynamic integration along pathways traversing this transition remains highly problematic.

Finally, we would like to point out that our derivation of the quasiequilibrium distribution for glassy systems is very widely applicable to any kind of aged polymorphic system. In general, the topology of ergodic phase space subdomains is far simpler for polymorphs than it is for glasses.

\section{ACKNOWLEDGMENTS}

We thank the National Computational Infrastructure (NCI) for computational facilities and the Australian Research Council (ARC) for funding.

\section{APPENDIX: CURVE FITTING FOR FOURIER TRANSFORMING THE CORRELATION FUNCTIONS}

The correlation functions were fitted using two different functions for short and long times. At shorter times we used

$$
C(t)=A+\frac{B}{1+C t^{D}}
$$

and at longer times we used

$$
C(t)=A \exp \left(-B t^{C}\right)+D \text {. }
$$

\footnotetext{
${ }^{1}$ P. W. Anderson, Science 267, 1615 (1995).

${ }^{2}$ P. G. Debenedetti and F. H. Stillinger, Nature (London) 410, 259 (2001). ${ }^{3}$ C. A. Angell, Science 267, 1924 (1995).

${ }^{4}$ P. G. Debenedetti, Metastable Liquids Concepts and Principles (Princeton University Press, Princeton, 1996).

${ }^{5}$ A. M. Puertas, E. Zaccarelli, and F. Sciortino, J. Phys.: Condens. Matter
} 
17, L271 (2005).

${ }^{6} \mathrm{~K}$. Binder and W. Kob, Glassy Materials and Disordered Solids (World Scientific, Singapore, 2005).

${ }^{7}$ F. Sciortino and P. Tartaglia, Adv. Phys. 54, 471 (2005).

${ }^{8}$ M. Reiner, Phys. Today 17 (1), 62 (1964).

${ }^{9}$ R. S. Lakes, Viscoelastic Solids (CRC, Boca Raton, 1998).

${ }^{10}$ J. C. Mauro, P. K. Gupta, and R. J. Loucks, J. Chem. Phys. 126, 184511 (2007).

${ }^{11}$ G. C. Boulougouris and D. N. Theodorou, J. Chem. Phys. 130, 044905 (2009).

${ }^{12}$ J. C. Mauro, D. C. Allan, and M. Potuzak, Phys. Rev. B 80, 094204 (2009).

${ }^{13}$ R. M. Christensen, Theory of Viscoelasticity (Dover, Mineola, NY, 1982).

${ }^{14}$ L. D. Landau and E. M. Lifshitz, Theory of Elasticity, 3rd ed. (Elsevier, Amsterdam, 1986).

${ }^{15}$ L. D. Landau and E. M. Lifshitz, Statistical Physics (Pergamon, Oxford, 1968).

${ }^{16}$ W. Voight, Ann. Phys. 283, 671 (1892).

${ }^{17}$ C. Zener, Phys. Rev. 52, 230 (1937).

${ }^{18}$ R. Lifshitz and M. C. Cross, Nonlinear Dynamics of Nanomechanical Resonators in Nonlinear Dynamics of Nanosystems, edited by G. Randons, B. Rumpf, and H. G. Schuster (Wiley-VCH, Weinheim, 2010).

${ }^{19}$ S. R. Williams and D. J. Evans, J. Chem. Phys. 131, 024115 (2009).
${ }^{20}$ D. J. Evans, D. J. Searles, and S. R. Williams, J. Stat. Mech.: Theory Exp. 2009, P07029.

${ }^{21}$ S. R. Williams and D. J. Evans, J. Chem. Phys. 127, 184101 (2007).

${ }^{22}$ R. G. Palmer, Adv. Phys. 31, 669 (1982).

${ }^{23}$ D. J. Evans and G. P. Morriss, Statistical Mechanics of Nonequilibrium Liquids, 2nd ed. (Cambridge University Press, Cambridge, 2008).

${ }^{24}$ D. J. Evans and D. J. Searles, Adv. Phys. 51, 1529 (2002).

${ }^{25}$ G. E. Crooks, J. Stat. Phys. 90, 1481 (1998).

${ }^{26}$ D. J. Evans, D. J. Searles, and S. R. Williams, J. Chem. Phys. 128, 014504 (2008).

${ }^{27}$ D. J. Evans, D. J. Searles, and S. R. Williams, J. Chem. Phys. 128, 249901 (2008).

${ }^{28}$ D. R. Squire, A. C. Holt, and W. G. Hoover, Physica (Amsterdam) 42, 388 (1969).

${ }^{29}$ W. G. Hoover, A. C. Holt, and D. R. Squire, Physica (Amsterdam) 44, 437 (1969).

${ }^{30}$ S. R. Williams and D. J. Evans, AIP Conf. Proc. 982, 74 (2008).

${ }^{31}$ S. Hess, M. Kroger, and W. G. Hoover, Physica A 239, 449 (1997).

${ }^{32}$ E. M. Sevick, R. Prabhakar, S. R. Williams, and D. J. Searles, Annu. Rev. Phys. Chem. 59, 603 (2008).

${ }^{33}$ S. R. Williams and D. J. Evans, Phys. Rev. E 78, 021119 (2008).

${ }^{34}$ S. R. Williams and D. J. Evans, Phys. Rev. Lett. 96, 015701 (2006).

${ }^{35}$ W. Kob and H. C. Andersen, Phys. Rev. E 51, 4626 (1995). 